Wir begrüßen kritische Auseinandersetzungen unserer Leser mit den in dieser Zeitschrift vorgetragenen Ansichten. Selbstverständlich kommt auch in ihnen lediglich die persönliche Meinung der Autoren zum Ausdruck.

Die Redaktion

\title{
NOCHMALS: \\ DIE TÜRKISCHE VERFASSUNGSREFORM VON 1971
}

\author{
Von KuRT RabL
}

Im Band 5 dieser Zeitschrift (S. 195 ff.) hat Ernst E. Hirsch über die Reform der 1961er Verfassungsurkunde der Türkischen Republik kurz berichtet und im Anschluß daran die deutsche Übersetzung nicht nur der Bestimmungen des einschlägigen Gesetzes Nr. 1488 v. 20./22. September 1971, sondern auch diejenige einiger, bereits vorher (kraft der Gesetze Nr. 1254/55 v. 17. April 1970 bzw. Nr. 1451 v. 30. Juni 1971) wirksam gewordener, weniger bedeutsamer Verfassungsänderungen mitgeteilt (a. a. O. S. 214 ff.). An diesem Bericht hat Bassam Tibi Kritik geübt (a. a. O. S. 447 ff.); seine Einwände richten sich sowohl gegen die Verfassungsänderung als solche als auch gegen Inhalt und Methode des Hirsch'schen Berichts. Sie sei im folgenden untersucht.

1. Beim Abdruck des Hirsch'schen Berichts hat sich die Redaktion aus Raumgründen gezwungen gesehen, auf eine Synopsis von altem und neuem Verfassungswortlaut zu verzichten. Sie konnte auf den Kommentar zur 1961er Verfassungsurkunde verweisen, den Hirsch vorgelegt hat und auf seinen - inzwischen erschienenen - Ergänzungsband, in dem die 1971er Verfassungsänderung übersetzt und kommentiert worden ist ${ }^{1}$. Für die Zwecke der folgenden Untersuchung läßt sich eine solche Gegenüberstellung indes nicht ganz vermeiden. Soweit es sich um die wichtigsten Einzelheiten handelt, sei sie im Nachfolgenden auf zwei der insgesamt 35 geänderten Verfassungsartikel beschränkt; in den anderen Fällen müssen können wohl auch - ein paar ganz allgemeine Hinweise genügen.

a) $\mathrm{Zu}$ betrachten ist zunächst Art. 11 der Verfassung. Er ähnelt dem Art. 19 Abs. 1-2 des Bonner Grundgesetzes und läßt Grundrechtseinschränkungen ausschließlich nach Maßgabe von Sinn und Wortlaut der Verfassung und nur in Form eines Gesetzes zu (Abs. 1); ferner wird wie in Art. 19 Abs. 2 BGG zwischen grundrechtseinschränkenden und solchen Gesetzen unterschieden, die ein Grundrecht in seinem Kern (Art. 19 Abs. 2 BGG: „Wesensgehalt") antasten - jene sind unter Wahrung der vorerwähnten Inhalts- und Formerfordernisse zulässig, diese niemals. Im Art. 19 BGG steht die Wesensgehaltsgarantie für sich allein; im Art. 11 der türkischen 1961er Verfassung war ihr ein erläuternder Zusatz angefügt, wonach dem Gesetzgeber auch dann verboten sein sollte, ein Grundrecht „,in seinem Kern anzutasten“, wenn dies „im Hinblick auf das öffentliche Wohl, die allgemeinen Sitten, die öffentliche Ordnung, die soziale Gerechtigkeit, die nationale Sicherheit oder aus ähnlichen Gründen" geschehen sollte (Abs. 2).

1 vgl. Ernst E. Hirsch, Die Verfassung der türkischen Republik (Die Staatsverfassungen der Welt in Einzelausgaben, Bd. 7), Frankfurt M. 1966; ders., Verfassungsänderung in der Türkei 1971 - Ergänzungsband (Die Staatsverfassungen der Welt in Einzelausgaben, Bd. 7a), Hamburg 1973. Was die neueste Verfassungsänderung (Ges. Nr. 1966 v. 15. März 1973) betrifft, so wird sie - ebenfalls in einer von Ernst E. Hirsch gefertigten Ubersetzung - im Anschluß an die vorliegende Untersuchung kommentarlos abgedruckt. Eine gelegentliche Erläuterung bleibt vorbehalten. 
Dieser Zusatz ist 1971 gestrichen worden. Dafür wurden drei Erweiterungen vorgenommen: (1) wurde in Abs. 1 eine der Sache nach einschränkende Zweckbestimmung eingeschoben; nach Maßgabe von Sinn und Wortlaut der Verfassung durch Gesetz angeordnete Grundrechtseinschränkungen sollten ihre Grenze nicht nur negativ in der vorerwähnten „Wesenskerngarantie“ finden (die aufrechterhalten wurde - Abs. 2 n. F.), sondern wurden überdies positiv an eine Reihe alternativ formulierter Voraussetzungen geknüpft, und zwar sollten sie nur „zum Schutz des Bestandes von Staatsgebiet und Staatsvolk, der republikanischen Staatsform, der nationalen Sicherheit, der öffentlichen Ordnung, des öffentlichen Wohls, der allgemeinen Sitten und der allgemeinen Gesundheit oder aus den in anderen Bestimmungen der Verfassung aufgeführten Gründen“ zulässig sein; (2) als neuer Absatz 3 wurde nach dem Vorbild der Art. 29 und 30 der Allgemeinen UNO-Erklärung über die Menschenrechte und Grundfreiheiten² und des Art. 17 des Europäischen Abkommens zum Schutz der Menschenrechte und Grundfreiheiten ${ }^{3}$, das auch für die Türkei gilt, eine Mißbrauchsklausel angefügt ${ }^{4}$; (3) als neuer Absatz 4 erscheint die Bestimmung, wonach, wie dies dem Geist des Art. 7 des Europäischen Abkommens entspricht, einschlägige Strafvorschriften nur in Form von Gesetzen in Kraft gesetzt werden dürfen.

b) Geändert worden ist ferner Art. 22 über die Pressefreiheit und die Freiheit, sich zu unterrichten. Absatz 1-3 dieses Artikels entsprach dem Art. 10 des Europäischen Abkommens, der seinerseits aus Art. 19 der Allgemeinen UNO-Erklärung abzuleiten ist, jedoch ging die türkische Verfassungsregelung von 1961 weniger weit als Art. 10: nach Art. 22 Abs. 3 der 1961er türkischen Verfassung bestand die Möglichkeit der Einschränkung der Pressefreiheit nur zum Schutz der Staatssicherheit (Art. 10: national security), der allgemeinen Sitten, der öffentlichen Ordnung (public safety), zur Verhütung von Angriffen gegen die Würde, Ehre und Rechte der Person, im Interesse der Verbrechensverhütung und zur Sicherung einer geordneten Rechtspflege, während Art. 10 außer diesen Fällen auch noch den Schutz der Unversehrtheit des Staatsgebiets, die Geheimhaltung vertraulicher Nachrichten, die öffentliche Gesundheit sowie alle anderen Fälle erwähnt, in denen Einschränkungen der Pressefreiheit ,in einer demokratischen Gesellschaft erforderlich" (necessary in a democratic society) sind.

Die Neufassung von 1971 hat einmal die Unversehrtheit des Staatsgebietes in die Reihe der in diesem Sinn geschützten Rechtsgüter aufgenommen, zum anderen die Geheimhaltung vertraulicher Nachrichten, jedoch nur insoweit, als es sich um Nachrichten handelt, die aus Gründen der Staatssicherheit geheimzuhalten sind. Der durch den Art. 10 des Europa-Abkommens gezogene Rahmen ist also nicht voll ausgefüllt. Hingegen ist ein im Art. 10 nicht ausdrücklich genannter Begriff neu eingeführt worden, und zwar derjenige der Unversehrtheit des Staatsvolkes, nicht nur des Gebietes.

Eine weitere wichtige Neuerung ist die in Art. 22 Abs. 5 n. F. eröffnete Möglichkeit der Zeitungsbeschlagnahme durch die Verwaltungsbehörden; jede solche Maßnahme ist jedoch binnen 24 Stunden dem Gericht mitzuteilen, das sie binnen

\footnotetext{
2 vgl. die UNO-Vollvers.-Entschließg. v. 10. Dez. 1948, Nr. 217-III.

3 v. 4. Nov. 1950, in Kraft seit 3. Sept. 1953; für die Türkei verbindlich seit 18. Mai 1954 (Hinterlegung der Ratifikationsurkunde beim Generalsekretär des Europarates).

4 Wortlaut des Abs. 3 s. u. zu Pkt. 3a des vorliegenden Berichts. Art. 17 des Europ. Abkommens lautet: „Nichts in diesem Abkommen darf dahin ausgelegt werden, als sei irgendeinem Staat, einer Gruppe oder Person das Recht eingeräumt, sich irgendeiner Tätigkeit zu widmen oder irgendeine Handlung mit dem Ziel vorzunehmen, die darin niedergelegten Rechte und Freiheiten zu zerstören oder in größerem Maß einzuschränken, als dies im Abkommen vorgesehen ist."
} 
dreier Tage ausdrücklich gutheißen muß, widrigenfalls sie „als nichtig angesehen“ wird. - Schon nach der 1961er Verfassung konnte das weitere Erscheinen von in der Türkei hergestellten Zeitungen und Zeitschriften durch Gerichtsurteil verboten werden, wenn sie „den auf den Menschenrechten und -freiheiten beruhenden Idealen der demokratischen und laizistischen Republik und der Grundnorm der Unteilbarkeit des Staatsgebiets und des Staatsvolkes" nicht entsprachen (Art. 22 Abs. 6 in Verb. mit Art. 57 Abs. 1). Nunmehr sind als Verbotstatbestände auch Verstöße gegen die Staatssicherheit, die öffentliche Ordnung und die allgemeinen Sitten hinzugekommen (Art. 22 Abs. 6 n. F.). Nach wie vor dürfen nur die Gerichte insoweit tätig werden. Es ändert sich auch nichts an der Möglichkeit, gemäß Art. 114 Abs. 3 für den Fall vom Staat Schadensersatz zu verlangen, daß das Gericht eine Beschlagnahmeverfügung "als nichtig ansieht".

c) Die übrigen Änderungen betreffen den Schutz des Privatlebens (Art. 15), die Religionsfreiheit (Art. 19), die Benützung nichtpressemäßiger Nachrichtenmittel (Art. 26), das Vereinsrecht (Art. 29 und 46), die persönliche Freiheit (Art. 30), die Gewähr des gesetzlichen Richters (Art. 32), die allgemeine Dienstpflicht (Art. 60) und die Steuerpflicht (Art. 61). In keinem Fall handelt es sich um die Aufhebung von Grundrechten, obwohl die Voraussetzungen für die Inanspruchnahme in zwei Fällen (Art. 26 - Recht auf Rundfunkempfang; Art. 29 - Vereinsrecht) eingeschränkt werden, wobei die durch die Art. 10 und 11 des Europa-Abkommens gezogenen Schranken jedoch gewahrt geblieben sind ${ }^{5}$. In einem anderen Fall (Art. 15 - Schutz des Privatlebens) wurde der Grundrechtsschutz verstärkt ${ }^{6}$. Abgeschwächt worden ist er hingegen im Fall der Landenteignung aus Gründen des öffentlichen Wohls (Art. 38); als Berechnungsgrundlage für den Entschädigungsbetrag galt nämlich früher (Abs. 1 a. F.) der Verkehrswert („wahrer Wert"), während nunmehr (Abs. 2 n. F.) der vom Eigentümer zuvor für Besteuerungszwecke angegebene Wert zugrunde zu legen ist, was dem Gedanken der sozialen Gerechtigkeit entsprechen dürfte.

2. Hirsch hat die Reform zusammenfassend dahin gekennzeichnet, daß es sich darum gehandelt habe, "die Strukturschwächen in der Verfassungsurkunde zu beseitigen und der Freiheit zur Zerstörung der Freiheit zu begegnen“ (a. a. O. S. 195). Er hat insbesondere darauf verwiesen, daß nicht der von der Armee veranlaßte Regierungswechsel am 12. März 1971 die Verfassungsreform ausgelöst habe, sondern die Tatsache, daß Gewaltverbrechen, politischer Terror und schwere Unruhen trotz des von der neuen Regierung angekündigten weitreichenden Reformprogramms fortgesetzt worden seien. Konnte man vorher sagen, daß jene Widerstandshandlungen im Geist des Vorspruchs zur 1961er Verfassungsurkunde - worin die Ausübung des Widerstandsrechts gegen eine, zufolge Verfassungs- und Gesetzesbruchs illegitim gewordene Obrigkeit ausdrücklich als verfassungsbegründender

\footnotetext{
5 Art. 10 Abs. 2: „Die Ausübung dieser Freiheiten (Meinung, Unterrichtung) kann, da sie Pflichten und Verantwortlichkeiten mit sich bringt, an Formerfordernisse, Bedingungen, Einschränkungen und Strafdrohungen geknüpft werden, soweit diese gesetzlich vorgeschrieben und in einer demokratischen Gesellschaft im Interesse der Staatssicherheit, Unversehrtheit des Gebiets, öffentlichen Ordnung, Verbrechensverhütung, zum Schutz von Gesundheit oder Sittlichkeit, des guten Rufes oder der Rechte anderer, zwecks Geheimhaltung vertraulicher Nachrichten oder zur Sicherung einer geordneten Rechtspflege erforderlich sind.“ Art. 11 tung vertraulicher Nachrichten oder zur Sicherung einer geordneten Rechtspflege erforderlich sind.“ Art. 11 geschränkt werden, es sei denn durch gesetzliche Vorschrift und soweit dies in einer demokratischen Gesellschaft im Interesse der Staatssicherheit oder der öffentlichen Ordnung, der Verbrechensverhütung, zum Schutz der Gesundheit oder Sittlichkeit oder der Rechte und Freiheiten anderer erforderlich ist. ${ }^{\alpha}$

6 Art. 15 Abs. 2 a. F. schützte eine Person, ihre Privatpapiere und persönlichen Sachen nur vor Durchsuchungen, es sei denn, daß sie in einem gesetzlich vorgesehenen Fall von der zuständigen Behörde angeordnet worden waren. Nunmehr ist dies nur noch zulässig, wenn die Angelegenheit keinen Aufschub duldet; außerdem muß im Regelfall ein richterlicher Durchsuchungsbefehl vorliegen. Ferner ist nunmehr auch die Beschlagnahme entsprechend geregelt.
} 
Akt legitimiert wird - als Versuch zu rechtfertigen waren, die Einleitung und Durchführung der lt. des Streitkräfte-Memorandums vom 12. März 1971 „in der Verfassung vorgesehenen Reformen" $z u$ erzwingen, so schien jetzt die Absicht deutlich zu werden, eben dies mit den gleichen Mitteln zu verhindern. Angesichts dessen stellte sich die Frage, ob und ggf. wie sich die verfassungsmäßige Ordnung gegen einen solchen Druck abschirmen ließ, und Hirsch bemerkt in diesem Zusammenhang, daß „sich auch hier wieder das nicht auf die Türkei beschränkte soziale Phänomen (zeige), daß eine freiheitliche demokratische Verfassung ,wegen ihrer Offenheit und ihrer mannigfachen Gewährleistungen und Freiheiten auch eine gefährdete (sic!) Ordnung' (sei), weil sie diejenigen politischen Gruppen, die ihre obersten Grundsätze und ihre Spielregeln prinzipiell verneinen, zum Mißbrauch der Freiheiten zwecks Beseitigung der Freiheiten aller anderen geradezu einlädt, wenn nicht sachgerechte Vorschriften gegen den Mißbrauch der Freiheiten vorhanden sind und angewandt werden" (a. a. O.).

3. Hiergegen wendet sich Tibi; er meint, daß die Verfassungsänderung deshalb erfolgt sei, „um die liberaldemokratischen Rechte schlechthin zu eliminieren“ (a. a. O. S. 453 f.), ja: daß durch sie die in der 1961er Verfassung "garantierten demokratischen Freiheiten und Grundrechte aufgehoben wurden“ (a. a. O. S. 447). Diese beiden Behauptungen widersprechen einander: denn wenn jene Rechte durch die Verfassungsänderung bereits aufgehoben wurden, brauchen keine Maßnahmen getroffen zu werden, um sie zu beseitigen - doch soll dies nicht vertieft werden. Tibi unternimmt, seine Meinung mit Zitaten aus dem Hirsch'schen Bericht zu belegen; dabei handelt es sich sowohl im Zitate aus den neuen Verfassungsbestimmungen als auch um ein Zitat aus dem Bericht selbst. Der Nachprüfung halten sie freilich in keinem Fall stand.

a) Tibi zitiert aus den Neufassungen der Art. 11 und 22, wobei einmal die tatbestandsmäßigen Voraussetzungen nicht erwähnt werden, von denen die Zulässigkeit grundrechtseinschränkender behördlicher Eingriffe abhängig gemacht worden ist wie etwa die Formel „... allein durch Gesetz und nur nach Maßgabe des Wortlautes und Sinnes der Verfassung " in Art. 11 Abs. 1 n. F. - und die, rechts- und verwaltungstechnisch gesehen, Ermessensschranken für die Behörden darstellen; man kann Art. 11 Abs. 1 n. F. zulässigerweise nicht so zitieren, als erlaube er jede beliebige Freiheitsbeschränkung nach freiem Ermessen der Behörden. Entsprechend steht es mit dem Zitat aus Art. 22 Abs. 6; es genügt nicht, anzuführen, daß Zeitungen und Zeitschriften verboten werden können, ohne die verfassungsgesetzlich festgelegten Voraussetzungen dafür - u. a. Strafbarkeit des Inhalts nach Maßgabe des nullapoena-Prinzips, Gerichtsbeschluß, ggf. Amtshaftung für gerichtlicherseits „nichtig“ erklärte polizeiliche Beschlagnahmeverfügungen - zu erwähnen. Dies gilt um so mehr, als es gerade jene tatbestandsmäßigen Voraussetzungen sind, die - wie bereits erwähnt - die fraglichen Vorschriften in Gleichklang mit den im Europäischen Menschenrechtsabkommen enthaltenen Richtlinien gebracht haben.

Ein anderes Zitat ist aus positivrechtlicher Sicht verfehlt. Tibi führt die dem Europäischen Abkommen nachgebildete Mißbrauchsverbotsklausel des Art. 11 Abs. 3 in folgender Form an:

„Keines der in dieser Verfassung aufgeführten Grund- und Freiheitsrechte darf in der Absicht gebraucht werden, die Menschenrechte und -freiheiten oder den Bestand des türkischen Staatsgebiets und Staatsvolks oder die republikanische Staatsform mit ihren in der Verfassung festgelegten Wesensmerkma- 
len unter Berufung auf die Verschiedenheit von Sprache, Rasse, Klasse, Religion und Konfession zu beseitigen."

Es handelt sich um drei verschiedene Auslassungen: (1) die Einreihung der Menschenrechte und -freiheiten anderer unter die vor Mißbrauch (im Sinn der in Rede stehenden Vorschrift) geschützten Rechtsgüter; (2) die Worte „mit ihren in der Verfassung festgelegten Wesensmerkmalen“, womit der Begriff der republikanischen Staatsform eine ganz bestimmte Kennzeichnung erfährt und endlich (3) die Worte „unter Berufung auf die Verschiedenheit von Sprache, Rasse, Klasse, Religion oder Konfession", die grammatisch gleichermaßen zu allen drei, von der Vorschrift erwähnten Schutzgütern - Menschen- und Freiheitsrechte, Staatsgebiet und -volk, republikanische Staatsform mit ihren verfassungsmäßigen Wesensmerkmalen - gehören.

$\mathrm{Zu}$ 1) braucht im vorliegenden Zusammenhang nichts gesagt zu werden, wenngleich auffällig sein mag, daß Tibi die Menschenrechte und Grundfreiheiten auch bei seinem Zitat aus Art. 22 Abs. 6 nicht erwähnt.

$\mathrm{Zu} 2$ ) ist wichtig, sich zu vergegenwärtigen, daß sich der Mißbrauch staatsbürgerlicher Freiheitsrechte nicht nur gegen die republikanische Staatsform als solche, sondern gegen ihre besonderen verfassungsmäßigen Kennzeichen richten muß, um dem verfassungsgesetzlichen Verbot des Art. 11 Abs. 3 zu unterfallen. Die Kennzeichen des türkischen Republikanismus faßt Art. 2 der Verfassung, der unverändert geblieben ist, in die Formel: „ein auf den Menschenrechten und den im Vorspruch zum Ausdruck kommenden Grundsätzen ruhender nationaler, demokratischer, laizistischer und sozialer Rechtsstaat"; die im Vorspruch zum Ausdruck kommenden Grundsätze lassen sich folgendermaßen umschreiben: gesinnugsmäßige Einung aller Glieder der türkischen Nation, Volkssouveränität, Bindung an die Reformen Atatürks, Verwirklichung und Sicherung der Menschenrechte und Grundfreiheiten, nationale Solidarität, soziale Gerechtigkeit, Sicherheit und Wohlfahrt des einzelnen und der Gesamtheit. Wenn die Verfassung verbietet, ein Grund- oder Freiheitsrecht in der Absicht zu gebrauchen, die republikanische Staatsform zu beseitigen, so kann dieses Verbot nur dann rechtlich zutreffend begriffen werden, wenn diese kennzeichnenden Merkmale des türkischen Republikanismus ebenfalls in den Blick genommen werden; man kann Art. 11 Abs. 3 daher zulässigerweise nicht so zitieren, wie es hier geschieht.

$\mathrm{Zu}$ 3) muß man sich klarmachen, daß die Mißbrauchsschutzklausel des Art. 11 Abs. 3 weniger weit geht als der zugrunde liegende Art. 17 des Europäischen Abkommens. Während dieses nämlich jeden Akt und jede Tätigkeit ausschließen will, die in irgendeiner Weise auf die Zerstörung oder unzulässige Einschränkung der im Abkommen gewährleisteten Freiheiten gerichtet ist, stellt Art. 11 Abs. 3 einerseits auf den Gebrauch von Grund- und Freiheitsrechten zum Schaden gleicher Rechte von Mitbürgern oder zum Schaden des Staatsganzen, andererseits darauf $a b$, daß dieser polemisch-agitatorische Gebrauch jener Rechte unter ausdrücklicher Berufung auf die Verschiedenheit der im Abs. 3 genannten Bevölkerungsschichten erfolgt. Erst dann wird der Gebrauch von Grund- und Freiheitsrechten im Sinn dieses Absatzes zum verfassungsgesetzlich verpönten Mißbrauch. Es handelt sich also darum, zu verhüten, daß, etwa anknüpfend an die Verfehlungen oder das Ungenügen einzelner, eine ganze Bevölkerungsschicht — „die“ Kurden, „die“ Juden, „die“ Reichen, „die“ Frommen, „die“ Gottlosen - zur Zielscheibe von Hetze wird in der Absicht oder auch nur mit der Folge, die ganze Gruppe kollektiv zu diskriminieren und dadurch das Staatsganze zu gefährden. 
Diese Einschränkung, die zugleich den staatsideologischen Grundzug der Verfassung überaus kennzeichnend zum Ausdruck bringt, darf nicht übersehen werden; es genügt nicht, lediglich anzuführen, daß die Grund- und Freiheitsrechte nicht gebraucht werden dürfen, um den Bestand des Staatsgebiets und -volkes oder die republikanische Staatsform zu beseitigen. Man kann Art. 11 Abs. 3 n. F. zulässigerweise nicht so zitieren.

b) Tibi beschuldigt Hirsch, dieser habe die 1961er Verfassung als "gefährliche (sic!) Ordnung" bezeichnet, „zumal sie zum Mißbrauch der Freiheiten aller anderen geradezu einlädt“ (a. a. O. S. 448). Dieses Zitat hat zwei Fehler: einmal wird ausgelassen, wen nach Hirsch' Worten eine rechtsstaatlich-freiheitliche Ordnung zum Mißbrauch der von ihr gewährleisteten Freiheiten einlädt - nicht jedermann (wie man nach Tibis Zitierweise glauben könnte), sondern diejenigen Gruppen, die die obersten Grundsätze und Spielregeln, nach denen allein eine solche Ordnung gehandhabt werden kann, prinzipiell verneinen. Zum anderen wird Hirsch' Kennzeichnung der rechtsstaatlich-freiheitlichen Ordnung als zufolge ihrer Offenheit stets gefährdet durch die Wiedergabe des Wortes "gefährdet" mit "gefährlich“ in ihr Gegenteil verkehrt.

c) Ergebnis: Die Behauptung, daß die den türkischen Staatsbürgern kraft der 1961er Verfassung gewährleisteten Menschenrechte und Grundfreiheiten durch die Verfassungsreform von 1971 "aufgehoben“ worden seien, läßt sich — zumindest fürs positive Recht der beiden, vorstehend erörterten Art. 11 und 21 - nicht aufrechterhalten. Ob die Behauptung richtig ist, daß die Urheber der Reform dahingehende Absichten hegten, kann an Hand der Tibischen Ausführungen, die in dieser Hinsicht kein schlüssiges Material beistellen, weder bewiesen noch bestritten werden. Eine etwaige Klärung dieser Frage wäre juristisch indes belanglos, es sei denn, daß erwiesen werden könnte, inwieweit diese Absicht auf Sinn und Wortlaut der neuen Bestimmungen entscheidend von Einfluß gewesen ist.

Umgekehrt spricht das Streben, die Wirksamkeit der in ihrem Wesen rechtsstaatlich-freiheitlich und demokratisch orientierten Staatsidee, wie sie von Kemal Atatürk überkommen ist, unangetastet $\mathrm{zu}$ lassen und $\mathrm{zu}$ sichern und insbesondere auch die Anlehnung der Novelle an einschlägige Bestimmungen des Europäischen Abkommens - die vorangegangenen Hinweise enthalten Belege für beides — für die Richtigkeit des analytischen Urteils, das Hirsch abgegeben hat.

4. Der zweite Einwand, den Tibi erhebt, richtet sich gegen die von Hirsch angewandte wissenschaftliche Methode. Er vernachlässige sowohl in seinem Bericht als auch in seinem 1966 veröffentlichten Standardwerk über die türkische Verfassung den "gesellschaftlichen Kontext", in dem Verfassungen stehen, er lasse insbesondere die gesellschaftlichen und historischen Grundlagen der türkischen Verfassungsentwicklung unberücksichtigt und befasse sich mit der 1971er Verfassungsreform „nur formal-juristisch“, mehr noch: er "unterschlage“ (sic!) den Zusammenhang von Gesellschaft, Politik und Verfassung (a. a. O. S. 449). Dieser heftige, die Grenzen der Ehrenkränkung streifende Vorwurf scheint einerseits darauf zu beruhen, daß Hirsch' Ausführungen, die das Gegenteil beweisen, in Tibis Kritik keinen Widerhall gefunden haben, zum anderen auf einem methodologischen Mißverständnis.

a) $\mathrm{Da}$ die nur knapp drei Seiten lange Einleitung zur deutschen Übersetzung der 1971er Verfassungsreformbestimmungen sich darauf beschränken mußte, den Ablauf der äußeren Ereignisse zu skizzieren, aufgrund deren ihr Wortlaut vereinbart und verabschiedet wurde, sollte nicht strittig sein. Was hingegen das Hirsch'sche 
Handbuch betrifft, so setzt seine Darstellung mit den Worten ein, daß Bedeutung und Tragweite der 1971er Verfassung nur unter Berücksichtigung der die Verfassungswirklichkeit bestimmenden materiellen und immateriellen Faktoren angemessen begriffen werden können? ${ }^{7}$. Diese Faktoren werden sodann auf rund dreißig Seiten monographisch darzustellen versucht, wozu noch eine rund siebzig Seiten starke Dokumentation kommt ${ }^{8}$. Hirsch kennzeichnet die nach seiner Meinung grundlegenden türkischen verfassungsrechtlichen Kräfte und Grundsätze als die Thesen von der Rechtsnachfolge (juristische Identität) zwischen dem Osmanischen Reich und der Türkischen Republik, von der Beständigkeit (juristische Kontinuität) des demokratischen Gedankens innerhalb der türkischen Geistes- und Verfassungsgeschichte und von der juristisch-ethischen Legitimationsbedürftigkeit der Revolutionen von 1921/24 und 1961 und er schließt daran einerseits eine scharfe Kritik der Verfehlungen, die zum Verfassungsumschwung von 1960/61 geführt haben sowie eine Skizze des Aufbaues und Inhalts der neuen Verfassung. Es folgt ein längerer, „Die metaphysische (nb.! - K.R.) Garantie der verfassungsrechtlichen Ordnung “ überschriebener Abschnitt, der die geistig-kulturellen Mindesterfordernisse für das angemessene Funktionieren einer demokratisch-parlamentarischen Verfassung, die sozialpsychologischen Gefahren der Industrialisierung und Intellektualisierung, die Einbruchstellen der 1961er Verfassungsordnung für sozialrevolutionäre Ideen, das Problem der politischen Demagogie (an Hand eines längeren Zitats eines türkischen Verfassungsrechtlers) und die Rechtstellung der politischen Parteien behandelt. Diese Ausführungen erscheinen - gerade auch, wenn man sie heute rückschauend liest - geeignet, die tieferen politischen Ursachen der Ereignisse zu kennzeichnen, die zur Verfassungsänderung von 1971 und zur derzeitigen Krisensituation in der Türkei geführt haben. Gegen das dort Gesagte mag man vielleicht Einwände erheben. Wer dieser Meinung ist, hat die Möglichkeit, eine im einzelnen begründete Kritik zu üben. Nicht zulässig erscheint jedoch, die Behauptung aufzustellen, als habe Hirsch die gesellschaftlichen und historischen Grundlagen der türkischen Verfassungsentwicklung unberücksichtigt gelassen. Dergleichen ist nicht nur objektiv unrichtig, sondern auch geeignet, die Möglichkeit wissenschaftlichen Meinungsaustausches von vornherein zu verschütten. Noch weniger zu billigen ist, wenn ein Gedankengang, an dem Kritik geübt wird, falsch wiedergegeben und die Diskussion dadurch von Anfang an auf ein Gleis geschoben wird, auf das sie nicht gehört ${ }^{9}$.

b) Es herrscht kein Streit darüber, daß zutreffende Verfassungserkenntnis sowohl die Kenntnis der Bestimmungen der in Betracht kommenden Verfassungsurkunde und der daran anknüpfenden Praxis der politischen, administrativen und rechtsprechenden Verfassungsorgane als auch die Kenntnis der geschichtlich-gesellschaftlichen Verhältnisse voraussetzt, die durch jene Bestimmungen geordnet bzw. durch jene Praxis gestaltet werden sollen. In der Tat leitet Hirsch seinen Verfassungskommentar mit dem Hinweis auf eben diese, der Rechtswissenschaft geläufige Grunderkenntnis ein ${ }^{10}$. Allerdings wird die Rechtswissenschaft - nicht anders als die Gesellschaftslehre - stets von dem ihr gemäßen Wissensstoff auszugehen

\footnotetext{
7 s. Hirsch, Verfassung a. a. O. (s. o. Anm. 1) S. 22; ebenso Verfassungsänderung a. a. O. (s. o. Anm. 1) S. $8 \mathrm{ff}$.

8 s. Hirsch, Verfassung a. a. O. S. $23 \mathrm{ff}$. und $195 \mathrm{ff}$.

9 Man vergleiche z. B. Tibis Darstellung a. a. O. S. 449 (Text zu Anm. 16), andererseits die zugrundeliegenden Ausführungen Hirsch' a. a. O. S. 34 ff. (Ziff. 27/28). Hirsch gebraucht den Ausdruck "Parallele zu den politischen Verhältnissen .... in denen Militärputsche zum politischen Spiel gehören“ unter Bezugnahme auf einen ganz anderen Sachverhalt, wie Tibi angibt. Dies ist um so stoßender, als Tibi gleichzeitig den Vorwurf erhebt, Hirsch habe eine "unhaltbare" Behauptung aufgestellt.

10 a. a. O. S. 21 (Ziff. 1).
} 
haben und zu ihm zurückkehren müssen: der Jurist wird also in erster Linie nicht nach den geschichtlich-gesellschaftlichen Verhältnissen, sondern danach zu fragen haben, welche Rechtsvorschriften und -entscheidungen sie ordnen sollen und wie es mit ihrer Anwendung bzw. Durchführung bestellt ist. Der Soziologe muß entgegengesetzt vorgehen - vorausgesetzt, daß Rechtliches ihm erforschenswert erscheint. Beides ist legitim. Schwierigkeiten ergeben sich, wenn eine der beiden Wissenschaften - etwa die Soziologie - vermeint, daß sich die andere an ihren methodischen Erfordernissen und Ergebniserwartungen auszurichten habe - bei Gefahr, andernfalls den Vorwurf gewärtigen zu müssen, „zu keinen nennenswerten, ja sogar zu völlig falschen Resultaten" gelangt zu sein. Hier erscheint ein höflicher Ordnungsruf nicht ganz unangebracht - nicht zuletzt deshalb, weil der Anspruch, einseitig im Besitz des rechten Weges zur wissenschaftlichen Wahrheit zu sein, die sicher nötige und auch fruchtversprechende Zusammenarbeit der Vertreter verschiedener Wissenschaften hindern könnte. Konkret: es ist erfahrungsgemäß nicht so, daß das Recht die von ihm geordneten Gesellschaftsverhältnisse ausschließlich widerspiegelt - es gestaltet sie auch, und dementsprechend muß der Jurist zwar soziologisch denken können, umgekehrt aber der Soziologe sich bemühen, juristische Kategorien kennen und handhaben zu lernen und sie dort, wo es nötig ist, in seine Arbeit einbeziehen. Daß Hirsch dieser Forderung genügt, wird kein unvoreingenommener Betrachter bestreiten können ${ }^{11}$; demgegenüber beschleicht den Leser der gegen ihn gerichteten erregten Kritik öfters das Gefühl, als fehle dort die Einsicht, daß jene Forderung nicht zu Unrecht erhoben wird.

5. Der Verfasser möchte nicht mißverstanden werden. Selbstverständlich kann man nicht daran vorbeisehen, daß die türkische Praxis hinsichtlich des Schutzes der verfassungsmäßigen Ordnung sowie der inneren Ruhe und Sicherheit neuerdings unter dem Gesichtspunkt der Menschenrechte und Grundfreiheiten Gegenstand von Kritik, u. a. auch in der Beratenden Versammlung des Europarates geworden ist ${ }^{11 a}$. Freilich muß man sich Wesen und Richtung dieser Kritik klarmachen. Ihre Grundlage ist nicht das geltende türkische Verfassungsrecht, sondern das Europäische Abkommen; da sich das türkische Recht jedoch - insbesondere in der Fassung, die es 1971 erhalten hat - an dieses Abkommen in einigen Punkten eng anlehnt, ist es sinnvoll, jene Praxis auch am türkischen Recht zu messen. Gerade aber, wenn man das tun will und soll, erhellt das Abwegige von Behauptungen, wonach die nach dem 1961er türkischen Verfassungsgesetz gewährleisteten Freiheitsrechte seit 1971 „aufgehoben“ seien: wären sie es, würde jeder Versuch, jene Praxis unter dem eben erwähnten Gesichtspunkt zu bewerten, mangels positivrechtlichen Maßstabes sinnlos sein.

Die Vorwürfe, die der türkischen Regierung gemacht werden, hat ein britisches Mitglied der Beratenden Versammlung, Judd, am knappsten und genauesten zusammengefaßt ${ }^{12}$ : man habe notstandsrechtliche Freiheitseinschränkungen mit rückwirkender Kraft eingeführt, ferner seien Einschränkungen der Vereins-, Versammlungs-, Gewerkschafts- und persönlichen Freiheit über das zulässige Maß hinaus in

11 Hirsch gehört zu den wenigen Juristen, die, anknüpfend an Ehrlich und Nußbaum, der Rechtssoziologie eigene monographische Arbeiten gewidmet haben - s. Das Recht im sozialen Ordnungsgefüge - Beiträge zur Rechtssoziologie (Bd. 1 der Schriftenreihe des - übrigens von Hirsch im Jahre 1964 gegründeten Instituts für Rechtssoziologie und Rechtstatsachenforschung der Freien Universität Berlin), Berlin 1966; vgl. auch Rechtssoziologie im Rechtsunterricht, in: Festschr. f. Rudolf Reinhardt, Köln 1972, S. 437 ff.; s. ferner Hirsch-Rehbinder (Hrsg.), Studien und Materialien zur Rechtssoziologie (Sonderheft 11 der Kölner Zeitschr. f. Soziologie), Köln 1967 usw., usw.

11a zum Folgenden insbes. das Protokoll der 24. Sitzg. der Beratenden Versammlung des Europarates am 23. Okt. 1972 - off. recs. AS (24) CR 15 und 16.

12 s. AS-CR 15, S. $33 \mathrm{ff}$. 
Kraft gesetzt bzw. gehandhabt worden; endlich habe man das - nach dem Europäischen Abkommen auch in Notstandszeiten uneingeschränkt geltende Folterverbot nicht beachtet. Es handelt sich um Übertretungen der Art. 15, 7, 11, 5 und 3 des Europäischen Abkommens, denen die Bestimmungen der Art. 124, 33, 29, 28, 46, 30 und 14 Abs. 3 der türkischen Verfassungsurkunde entsprechen. Diese Anschuldigungen haben sich Abgeordnete der Beratenden Versammlung des Europarates aus Skandinavien und den Niederlanden zu eigen gemacht; von belgischer und schweizerischer Seite ist dem ebenso widersprochen worden wie seitens des türkischen Außenministers und eines türkischen Abgeordneten ${ }^{13}$.

Die Sache hat eine tatsächliche und eine rechtliche Seite.

a) In tatsächlicher Hinsicht steht Aussage gegen Aussage. Es wäre nötig, den Sachverhalt anhand rechtlich faßbarer Belege nachzuprüfen: Gesetze müßten auf ihre Verfassungsmäßigkeit, behördliche Maßnahmen und Gerichtsurteile auf ihre Gesetz- und Verfassungsmäßigkeit untersucht werden; dies hätte anhand des genauen Wortlauts der in Betracht kommenden schriftlichen Unterlagen bzw. einwandfrei zustande gekommener, vorzüglich eidesstattlicher Aussagen von Betroffenen, Zeugen und/oder Sachverständigen zu geschehen. Vielleicht kann diese Klärung durch den Untersuchungsausschuß erfolgen, dessen Einsetzung Anfang 1973 von einigen skandinavischen, britischen, niederländischen und österreichischen Mitgliedern der Beratenden Versammlung des Europarates beantragt worden ist ${ }^{14}$. Solange dieser Ausschuß weder gebildet worden ist ${ }^{15}$ noch seinen Bericht vorgelegt hat, werden Außenstehende mangels anderer Möglichkeit objektiver Information wohl gut tun, endgültige Urteile zu diesem Fragenbereich zurückzustellen ${ }^{\mathbf{1 6}}$.

13 s. die Erklärungen der Abgg. Dankert (Niederlande), Aasen (Norwegen) und Ahlmark (Schweden) - ASCR 15 S. $13 \mathrm{ff}$. und $27 \mathrm{ff}$. bzw. AS-CR $16 \mathrm{~S}$. $39 \mathrm{ff}$; andererseits die Erklärungen der Abgg. de Clierco (Belgien) und Reverdin (Schweiz) - AS-CR 15 S. 24 ff. und 38 ff.; von türkischer Seite Außenmin. Bayülken - AS-CR 16 S. 46 ff. und der Abg. Fezioglu - AS-CR 14 (Sitzg. v. 21. Okt. 1972) S. $68 \mathrm{ff}$. und AS-CR 15 S. $20 \mathrm{ff}$

14 vgl. Antrag Darling u. Gen. v. 23. Jàn. 1973 - CE-doc. 3251.

15 Die Rechtsgrundlage hierfür wäre nicht im Europa-Abkommen selbst, sondern in Art. 45 Abs. 2 der Geschäftsordnung der Beratenden Versammlung zu finden.

16 Die Internationale Juristenkommission, Genf, die dazu berufen und geeignet sein kann, bei der Wahrheitsfindung zu helfen, hat in $\mathrm{Nr}$. 9 ihrer Review (Dez. 1972) einen anonymen Kurzbericht (S. $16 \mathrm{f}$.) veröffentlicht, der u. a. auf die Frage der Rückwirkung strafrechtlicher Bestimmungen eingeht; er erscheint nicht frei von Voreingenommenheit (es ist z. B. nicht ohne weiteres einzusehen, warum ein akascheint nicht frei von Voreingenommenheit (es ist z. B. nicht ohne weiteres einzusehen, warum ein aka-
demischer Lehrer, der Generalsekretär des Arbeitgeberverbandes ist, lediglich deshalb ungeeignet sein soll, an einem gerichtlichen Gutachten über die von einem Kollegen verfaßte "Einführung in die Verfassung mitzuwirken). Diese Stellungnahme hat denn auch aus den Kreisen der Kommissionsmitglieder Kritik gefunden. Ferner hat die Kommission am 21. März 1973 den Bericht von Prof. Dr. Peter Noll, Ordinarius für Straf- und Strafverfahrensrecht an der Universität Zürich, veröffentlicht, der in ihrem und im Auftrag von Amnesty International erstellt worden ist. Der Berichterstatter hat sich zehn Tage lang in der Türkei aufgehalten und u. a. während eines Tages mit Hilfe zweier Dolmetscher dem militärgerichtlichen Verfahren gegen Angehörige der sog. Dev-Genç (revolutionäre Jugend) beigewohnt. Sein Bericht (hekt. Pressemitt., im Archiv der Abt. ausländ. öf. Recht u. Völkerrecht der Forschungsstelle für Außenpolitik und Völkerrecht, Univ. Mainz) gipfelt im wesentlichen in folgenden Punkten: (1) die Militärrichter seien zufolge der Novellierung der für sie geltenden Laufbahn- und Beförderungsvorschriften (Ges. v. 15. Juli 1972, Nr. 1402) nicht unabhängig - allerdings laufe gegen die neuen Bestimmungen eine Verfassungsbeschwerde; (2) die Untersuchungshaft werde oftmals unzulässig ausgedehnt, und die Verteidigungsmöglichkeiten Beschuldigter während der Haft entsprächen nicht dem Art. 5 Abs. 2 des Europ. Abk. zum Schutz der Menschenrechte und Grundfreiheiten (unverzügliche genau Mitteilung des Haftgrundes); (3) Untersuchungshäftlinge seien gefoltert worden, worüber Erlebnis- und Zeugenaussagen vorliegen und auch in der Zeitung "Yeni Ortam“ (Neue Mitte, Istanbul) berichtet worden sei (2. u. 3. Febr. 1973); seit dem 4. Febr. 1973 sei die Veröffentlichung solcher Berichte verboten; (4) die Offentlichkeit der Hauptverhandlung sei nicht gewährleistet; (5) die Beweisaufnahme in der Hauptverhandlung vollziehe sich korrekt, und die Angeklagten seien in ihrer Verteidigung nicht behindert. Der Bericht vertritt abschließend die Meinung, daß der Belagerungszustand gefahrlos aufgehoben werden könne. (Die Frage ist, ob ein solches Urteil von einem landes- und sprachunkundigen Beobachter nach zehntägiger Anwesenheit sachkundig gefällt werden kann.) $\mathrm{Zu}$ o. Pkt. (1) ist $\mathrm{zu}$ erfahren, daß das türkische Verfassungsgericht die fraglichen Bestimmungen inzwischen in der Tat als verfassungwidrig erklärt hat. Die derzeitige Rechtslage ergibt sich aus Art. 136 Abs. 3 der Verf. i. d. Fassg. d. Ges. v. 15./20. März 1973 (vgl. die dtsch. Ubersetzg. u. S. $233 \mathrm{f}$.). Die diesbezüglichen Angaben von Noll - s. seinen Aufsatz „Der Ausnahmezustand in der Türkei“ in Nr. 99 der Fernausg. der NZZ v. 11. April 1973 - sind daher nicht mehr richtig. Zu o. Pkt. (3) vgl. Roth-Heinrich, Partner Türkei oder Unterdrückung für die Freiheit des Westens? Hamburg 1973 (rororoaktuell), S. $110 \mathrm{ff}$. (konnte vom Verf. nicht eingesehen werden), und auch der angekündigte Bericht des niederländ. Abg. der Beratenden Versammlung des Europarates, Dankert - s. Report on a fact-finding (private) mission to Turkey from 28th February - 10th March $1973-$, den er der Versammlung gegen 
b) Was hingegen die rechtliche Seite angeht, so haben die Verhandlungen der Beratenden Versammlung schon jetzt einen erörterungsfähigen Gesichtspunkt geliefert, der abschließend nicht zuletzt deshalb kurz gewürdigt werden mag, weil er nicht nur für den Einzelfall, sondern grundsätzlich wichtig zu sein scheint. Es handelt sich um das Verbot der Türkischen Arbeiterpartei durch das Verfassungsgericht und die sich in diesem Zusammenhang ergebenden Fragen der Auslegung des Art. 141 des türkischen Strafgesetzbuchs in Verbindung mit Art. 57 der Verfassungsurkunde (der 1971 nicht geändert wurde) und dem Gesetz vom 13. Juli 1965, Nr. 648, über die politischen Parteien ${ }^{17}$. Man hat der türkischen Praxis im Europarat vorgeworfen, sie dulde keine politischen Parteien „auf der Grundlage von Klassen“, obwohl solche Parteien in Westeuropa Regierungsverantwortung trügen ${ }^{18}$, und sie bestrafe die Führer solcher Parteien aufgrund einer Vorschrift, die so unbestimmt gefaßt sei, daß sie zur Unterdrückung jeder Opposition verwendet werden könne ${ }^{19}$.

Nach Art. 1 des türkischen Parteiengesetzes handelt es sich bei politischen Parteien um Vereinigungen, die durch Teilnahme an der Arbeit im Parlament und in den kommunalen Vertretungskörperschaften die Staats- und Gesellschaftsordnung mitbestimmen und die Tätigkeit der öffentlichen Organe beeinflussen und beaufsichtigen wollen und dies als fortwährende Aufgabe betrachten; zu diesem Zweck versuchen sie, bei den Wahlen ins Parlament und in die kommunalen Vertretungskörperschaften einen diesbezüglichen Auftrag in Form möglichst zahlenstarker Zustimmung zu ihrem Programm zu erhalten und haben das Recht, sich zu diesem Behuf frei $z u$ organisieren und frei für ihr Programm zu werben ${ }^{20}$. Unter dem Gesichtspunkt der Grundrechte und ihrer unbehelligten Inanspruchnahme kommt es also darauf an, ob eine Partei sich frei organisieren und frei für ihr Programm werben kann. Die Frage ist, ob diese Freiheiten sich auch auf die Aufstellung des Programms erstrecken oder ob für seinen Inhalt vom positiven Verfassungsrecht Grenzen gezogen sein können bzw. im türkischen Fall gezogen sind. Bestehen solche Grenzen nach positivem Verfassungsrecht, so folgt, daß die Organisations- und Werbefreiheit der politischen Parteien - und damit Meinungsfreiheit ihrer Führer und anderen Mitglieder - den damit gegebenen verfassungsrechtlichen Einschränkungen ebenfalls unterliegen.

Für die Bundesrepublik z. B. ergeben sich Grenzen solcher Art insbesondere aus den Art. 18, 20 (Abs. 3 Halbsatz 1, Abs. 4), 24 (Abs. 2), 25, 26 und 79 (Abs. 3) GG. Versucht man, die rechtliche Tragweite dieser Einschränkungen mit einer allgemeinen Formel zu umschreiben, so ließe sich sagen, daß Parteiprogramme so weit nicht aber weiter - gehen dürfen, als der Verfassungsgesetzgeber nach dem positiven Recht des GG gehen könnte; ein Parteiprogramm kann also Änderungen der staatlichen und gesellschaftlichen Ordnung der Bundesrepublik auch dann befür-

Ende März 1973 vorgelegt hat (Hinweis s. NZZ Fernausg. Nr. 81 v. 24. März 1973 - hekt. Abdruck im Archiv der Abt. ausländ. öff. Recht und Völkerrecht der Forschungsstelle für Außenpolitik und Völkerrecht, Univ. Mainz). Der Bericht behandelt u. a. die Voraussetzungen für die Verhängung und Aufrechterhaltung des Ausnahmezustandes, die Frage der Folterungen vorläufig Festgenommener vor ihrer Uberstellung in gerichtliche Untersuchungshaft sowie Verfahrensmängel der Militärstrafgerichte. (Er geht allerdings insoweit von falschen Voraussetzungen aus, als nicht ${ }^{2}$ der größte Teil der Türkei unter Ausnahmerecht steht ${ }^{\prime \prime}$ - a. a. O. S. $4-$, sondern das Standrecht für 7 von insges. 67 Provinzen ausgerufen worden ist.) Es wäre zu wünschen, daß der im Text erwähnte Ausschuß seine Tätigkeit bald aufnehmen könnte, um die Lage unter Beizug der dort ebenfalls erwähnten Hilfsmittel umfassend abzuklären.

17 Wortlaut des türk. StGB s. Jeschek-Mattes (Hrsg.), die strafrechtlichen Staatsschutzbestimmungen des Auslands, 2. Aufl., Bonn, 1968, s. $384 \mathrm{ff}$. (S. 390); ausführl. Inhaltsangabe des Parteienges. s. Hirsch, Verfassung a. a. O. (a. o. Anm. 1) S. $124 \mathrm{ff}$.

18 so der (niederländ.) Abg. Dankert - s. AS-CR $15 \mathrm{~S} .14 \mathrm{ff}$.

19 so der (schwed.) Abg. Ahlmark - s. AS-CR 16 S. $40 \mathrm{ff}$.

20 vgl. dazu die Analyse des Parteienges. bei Hirsch, Verfassung a. a. O. (s. o. Anm. 17). 
worten, wenn seine Durchführung Verfassungsänderungen erfordern würde, jedoch liegt die Grenze dort, wohin auch der deutsche Verfassungsgesetzgeber nicht vorzudringen vermöchte. Was selbst ihm schlechtweg verwehrt ist, soll auch keine deutsche politische Partei anstreben dürfen: dies ist wohl der innere Sinn des letztlich maßgebenden Art. 21 Abs. 2 GG.

Auch das türkische Verfassungsrecht kennt solche Schranken, die gewisse Grundsätze und -werte gegenüber jedermann - und daher auch gegen ein allfällig gegengerichtetes Streben politischer Parteien - abzusichern bestimmt sind. Diese Schranken ergeben sich aus Art. 57 Abs. 1 Satz 2 der Verfassungsurkunde21. Demnach darf sich die Tätigkeit einer politischen Partei nicht gegen die verfassungsmäßigen Grundrechte und gegen die im Vorspruch und in den Art. 1, 2, 3 (Abs. 1), 4 (Abs. 3), 8 und insbesondere 922 der Verfassung niedergelegten politischen Grundsätze und -werte des türkischen Verfassungslebens richten. Was hier zum Vorschein kommt, ist der Gedanke, daß Freiheitsrechte - und der Anspruch politischer Parteien auf grundsätzlich unbehelligtes Wirken ist, genau gesehen, die Bündelung der Ansprüche auf Meinungsfreiheit ihrer Führer und anderen Mitglieder - nur dann sinnvoll gebraucht werden, wenn die ihnen begrifflich innewohnenden Verantwortlichkeiten und die damit gegebenen Schranken für den eigenen politischen Handlungs- und Wirkensspielraum nicht außer acht gelassen werden. Dieser Gedanke ist auch ins Europäische Abkommen zum Schutz der Menschenrechte und Grundfreiheiten eingegangen - kennzeichnenderweise in die Vorschrift über die Gewährleistung des Rechts auf Meinungsfreiheit und der Freiheit, sich zu unterrichten ${ }^{23}$; er hat sich aber auch in der Rechtsprechung des türkischen Verfassungsgerichts niedergeschlagen ${ }^{24}$.

In diesem Zusammenhang ist insbesondere auf Art. 4 Abs. 3 Satz 1 und auf den bereits mehrfach erwähnten Art. 11 Abs. 3 der türkischen Verfassung aufmerksam zu machen. Letzterer erklärt bekanntlich den Gebrauch von Freiheitsrechten in verfassungswidriger Absicht insbesondere dann zum Mißbrauch, wenn dies „unter Berufung auf die Verschiedenheit von Klasse(n)" geschieht, und ersterer verbietet, „die Ausübung der Staatsgewalt einer bestimmten Klasse zu überlassen“ eine Vorschrift, die aus dem Jahr 1961 stammt und von der 1971er Verfassungsänderung unberührt geblieben ist. Wenn in der Türkei also politische Parteien nicht geduldet werden, deren Programm und/oder deren Tätigkeit sich auf ver-

21 Art. 57: „Statuten, Programme und Tätigkeit der politischen Parteien müssen den auf den Menschenrechten und -freiheiten beruhenden Idealen der demokratischen und laizistischen Republik und der Grundnorm der Unteilbarkeit des Staatsgebiets und Staatsvolks entsprechen." Satz 2: "Parteien, die sich nicht danach richten, werden für immer verboten " - Ubersetzg. nach Hirsch, Verfassung a. a. O. (s. o. Anm. 1) S. 124.

22 Vorspruch zur 1961er Verfassungsurkunde: „Die Türkische Nation... (Abs. 3:) „begeistert und beseel vom türkischen Nationalismus, der alle Einzelnen - Schicksalsgenossen in Glanz und Elend - zu einem unteilbaren Ganzen um das nationale Bewußtsein und um die nationalen Ideale schart und das Ziel verfolgt, unsere Nation als gleichberechtigtes und geachtetes Mitglied der Völkerfamilie der Welt im Geist nationaler Einheit ständig zu erhöhen“, (Abs. 5:) „vom Wunsch geleitet, einen demokratischen Rechtsstaat mit allen seinen rechtlichen und sozialen Grundlagen zu errichten, der es ermöglicht, die Menschenrechte und -freiheiten, die nationale Solidarität, die soziale Gerechtigkeit, die persönliche Sicherheit und die Wohlfahrt des Einzelnen und der Gesamtheit zu verwirklichen und zu sichern . . .“ Art. 1: „Der türkische Staat ist eine Republik.“ Art. 2: "Die türkische Republik ist ein auf den Menschenrechten und den in der Präambel zum Ausdruck kommenden Grundprinzipien ruhender nationaler, demokratischer, laizistischer und sozialer Rechtsstaat.“ Art. 3 Abs. 1: "Der türkische Staat bildet mit seinem Gebiet und seinem Volk ein unteilbares Ganzes. “ Art. 4 Abs. 3: "Die Ausübung der Staatsgewalt darf in keiner Weise einer bestimmten Einzelprson, Gruppe oder Klasse überlassen werden. Keine Person und kein Organ darf eine Funktion des Staates wahrnehmen, die nicht ihre Quelle in der Verfassung findet.“ Art. 8 Abs. 1: "Gesetze dürfen nicht gegen die Verfassung verstoßen.“ Art. 9: „Die Verfassungsbestimmung über die Republik als Staatsform darf nicht geändert, ein Vorschlag auf Abänderung nicht eingebracht werden."

23 vgl. Art. 10 Abs. 2 (s. den. Wortlaut o. in Anm. 5); zu beachten ist der Einschub: "da sie (nl. die Ausübung dieser Freiheiten) Pflichten und Verantwortlichkeiten mit sich brinigt ${ }^{\text {". }}$

24 „Um ihre soziale Aufgabe erfüllen zu können, ist Voraussetzung, daß (das) Verantwortungsgefühl (der Presse) ebensoweit reicht wie ihre Freiheit. Eine Presse ohne Verantwortungsbewußtsein entartet früher oder später ebenso wie jeder verantwortungslose Mensch “ - so die von Hirsch, Verfassung a. a. O. (s. o. Anm. 1) nachgew. Entcheidung, amtl. Slg. Bd. 1 S. 159 ff. (S. 164). 
fassungswidrige Ziele ${ }^{25}$ mit der Begründung richtet, daß dies durch „die Verschiedenheit von Klasse(n) “ innerhalb der Staatsbevölkerung gerechtfertigt werden könne, so ist das verfassungsrechtlich nicht nur nicht $\mathrm{zu}$ beanstanden, sondern entspricht sogar einem positivrechtlichen Verfassungsgebot. Dasselbe Gebot vollziehen auch die Art. 141 und 142 des türkischen, bereits im Jahre 1926 erlassenen Strafgesetzbuchs, wonach die Bildung und Leitung von Vereinen mit dem Ziel, bzw. die Werbung für den Gedanken, „die Herrschaft einer sozialen Klasse über die andere zu errichten oder eine soziale Klasse zu unterdrücken oder die im Staat bestehenden wirtschaftlichen oder gesellschaftlichen Grundordnungen zu zerstören ", mit Strafe bedroht wird.

Fragt man nach dem begrifflichen Standpunkt und Sinn des damit zum Ausdruck kommenden Gedankens im Gesamtgefüge der türkischen Verfassungsordnung, so ergibt sich die Antwort möglicherweise im Rückgriff auf den bereits erwähnten Art. 11 Abs. 2: wird dort nämlich die rechtliche Unantastbarkeit des Wesenskerns der Grundrechte gegenüber etwaigen staatlichen Zugriffen gewährleistet, so hier die Unantastbarkeit des Wesenskerns der Verfassung gegenüber Zugriffen durch eine in diesem Sinn „unverantwortliche“ Inanspruchnahme von Grundrechten.

Damit kann die Betrachtung sich abschließend nochmals der Tibi'schen Kritik der 1971er Verfassungsreform zuwenden. Tibi glaubt feststellen zu können, daß die Reform „von vornherein eine legale, auf eine Systemtransformation hinarbeitende Opposition verbiete(.) (a. a. O. S. 448) und meint wohl, damit ein abwertendes Urteil abgegeben zu haben, dessen Richtigkeit gar nicht mehr nachgeprüft zu werden brauche. Indes wird hier die der türkischen Verfassung - nicht wesentlich anders als dem Bonner Grundgesetz - innewohnende Spannung zwischen den Polen des individuellen und des kollektiven Interesses, zwischen dem, zur Gewährleistung seiner Menschenrechte an den Staat verwiesenen Bürger und dem, zur Gewährleistung seiner Funktionsfähigkeit und -tüchtigkeit an die Loyalität des Bürgers verwiesenen Staat nicht richtig gesehen. Darüber hinaus wäre zu fragen, was unter dem näherer Kennzeichnung bedürftigen Ausdruck „System“ zu verstehen ist, das "transformiert" werden soll. Sind es die oben ${ }^{26}$ erwähnten, kraft positiven Verfassungsrechts niedergelegten Grundsätze und -werte des türkischen Verfassungslebens - wozu insbesondere auch das in den Art. 141 und 142 StGB enthaltene, seit bald einem halben Jahrhundert geltende Verbot der die Unteilbarkeit des türkischen Staatsvolkes zerstörenden Unterdrückung einer Klasse durch die andere gehört?

6. Dem Verfasser liegt nicht daran, die hier notgedrungen geübte „Anti-Kritik“ mit dieser Frage abzuschließen, die nach seinem Willen keinen polemischen Unterton, sondern lediglich die Bitte um Auskunft enthalten will. Ihm ist die Türkei aus einem mehrmonatigen wissenschaftlichen Studienaufenthalt ein wenig bekannt geworden. Zweierlei ist ihm durchgängig aufgefallen: auf der einen Seite der Lerneifer und Wissensdrang der jüngeren Generation, der keineswegs auf studentische Kreise beschränkt ist und aus der klaren Erkenntnis fließt, daß ein Volk, das in der modernen Welt einen modernen Staat besitzen will, selbst modern sein, d. h.

\footnotetext{
25 im Sinn des Art. 57 Abs. 1 Satz 2 in Verb. m. Art 1, 2, 3 (Abs. 1), 4 (Abs. 3 ), 8 und 9 der Verfassung s. o. Anm. 19 und 20. Nicht verboten ist die Propagierung der marxistischen Lehre vom Klassenkampf als solcher - vgl. dazu Hirsch, Verfassungsänderung a. a. O. (s. o. Anm. 1) S. 11.. Die Grenze liegt dort, wo unter Berufung auf diese Lehre dafür geworben werden würde, die Angehörigen einer bestimmten Gesellschaftsschicht der Bevölkerung unter Nichtbeachtung des Gleichheitsgrundsatzes (Art. 12 der Verfassung) in ihren Menschenrechten oder Grundfreiheiten zu beschneiden - s. Art. 141 und 142 des türk. StGB von 1926 (im Text hiernach).

26 s. Anm. 22.
} 
eine möglichst intensive, vielseitige Erziehung (Schulbildung und Erwachsenenbildung) genossen haben muß, weil allein dadurch jene Energien und Initiativen freigesetzt werden, deren ein modernes Wirtschaftsgefüge bedarf - auf der anderen Seite der unübersehbare Widerwille vieler junger türkischer Akademiker, nach Abschluß ihrer Studien als Lehrer, Ärzte, Verwaltungsbeamte oder in ähnlicher Stellung außerhalb der drei großen Städte des Landes tätig zu sein. Auch besteht in diesen Kreisen mitunter mehr Neigung, ins westliche Ausland zu gehen und dort $\mathrm{zu}$ verbleiben, als sich der sicher in vielem mühevollen und auch entsagungsreichen Aufgabe zu unterziehen, an jenem allgemeinen Werk der Volkserziehung - namentlich in Zentral- und Ostanatolien - mitzuwirken. Der freiheitliche Rechtsstaat kann seine jungen Bürger insoweit kaum einem $Z_{\text {wang unterwer- }}$ fen - diese sind es vielmehr in erster Linie selber, die es in der Hand haben, dafür zu sorgen, daß die Staatsbevölkerung insgesamt raschmöglich in den Stand gesetzt wird, sich aus eigener Kraft in jenen intellektuell-zivilisatorischen Standard hineinzuarbeiten, den die Verfassungsurkunde und das von ihr gewollte System des demokratisch-parlamentarischen, republikanisch-rechtsstaatlichen Mehrparteisystems für sie vorgezeichnet hat. 
rity could be punished without granting the regular legal procedure, served mainly this purpose.

Field-work, done in the Republic of Niger, shows that until now the effectiveness of official jurisdiction is still limited. The majority of civil and criminal cases are not brought to court, but are settled by chiefs, by the administration, or between the two parties involved.

This rather diffuse distribution of jurisdiction shows that to possess executive power is of little value. An independent jurisdiction exists only on paper. In the majority of the cases, the government asserts its claims directly - bypassing all official authority or, at the most, using them as a mere instrument.

\section{Once Again: Constitutional Reform in Turkey 1971}

\section{By Kurt Rabl}

In no. 2/1972 of this journal Ernst E. Hirsch has published a translation of the 1971 constitutional reform in Turkey. Commenting upon this reform, he explained that it has been necessary in order to safeguard the democratic and liberal structure of the country's system endangered by tendencies to overthrow the existing order by abusing the liberties it guarantees. This report has been criticized by B. Tibi in no. 4 of the year 1972. This author maintained that the 1971 reform in fact abrogated most of the political rights guaranteed in 1961 in order to prohibit social changes and to shift those into a position of constitutional illegality who are advocating them.

This thesis is challenged and refuted. Concentrating upon but two of the most outstanding examples (arts. 11 und 22 of the constitution in its new form as compared with its original text) it is demonstrated, especially by confronting the new wording with the European Convention for the Protection of Human Rights (to which Turkey is a party) as well as by comparison to the Bonn Basic Law, that this opinion cannot be upheld. In this connection it is pointed out that, according to Turkish as well as German positive constitutional law, any social or economic reform must respect certain basic rules, concepts and values which, in law, are protected by insurmountable barriers. As to Turkey, these are certain political principles laid down by Atatürk (parliamentarism, republicanism, secularism, human rights, rule of law etc.). On the other hand, it must not be overlooked that the practical application of certain liberties has been challenged recently from various quarters inside and outside Turkey, and these grievances have found reverberation also within the Consultative Assembly of the Council of Europe though they have been denied or doubted from the Turkish as well as from other quarters. This discrepancy ought to be solved by an impartial investigation, to be conducted by a special committee nominated by the Assembly, and a motion has already been tabled to this effect. It is to be hoped that the Assembly will soon act accordingly. 